\title{
STEROID-INDUCED T WAVE CHANGES IN HEART BLOCK
}

\author{
BY \\ CLIVE P. ABER AND E. WYN JONES \\ From the Heart Department, Liverpool Royal Infirmary; and the Liverpool Regional Cardiac Centre \\ Received May 13, 1964
}

Giant $T$ waves have previously been described by several observers. In most instances this electrocardiographic pattern has been associated either with acute myocardial infarction (Levine and Brown, 1929; Wood and Wolferth, 1934) or recent complete heart block (Ippolito, Blier, and Fox, 1954; Szilagyi and Solomon, 1959), and a slow ventricular rate is believed to be an important, but not invariable, determinant of this $T$ wave configuration (Garcia-Palmieri et al., 1956). Stretching of areas of focally damaged myocardium by diastolic overloading and variations in vagal tone have been suggested as possible causes of this electrocardiographic abnormality (Scherf, 1944; Ippolito et al., 1954; Szilagyi and Solomon, 1959) which could in fact represent disturbance of the electrolyte flux and sodium-potassium pump across the cell membrane. Satisfactory confirmation of these hypotheses is, however, still lacking.

We have made observations, during steroid treatment of patients with established heart block, which appear to support the concept that local ionic disturbances are responsible for this perversion of myocardial repolarization. As far as we are aware, steroid-induced T wave changes of this form have not previously been reported.

\section{Subjects AND Methods}

Between 1958 and 1963, 30 patients with either complete or partial atrio-ventricular block were treated with corticotrophin or corticosteroids in an attempt to restore sinus rhythm (Aber and Wyn Jones, 1960; and unpublished material). Electrocardiograms were obtained in all of these patients during this treatment.

Serum transaminase (SGOT) levels were determined before commencing treatment with steroids, and serum electrolytes were measured before and during the course of therapy.

Case 1. This 66-year-old woman had experienced exertional cardiac pain and repeated "blackouts" for one year before admission to hospital in September 1961. Clinical examination revealed bradycardia (36/min.) and evidence of calcific aortic stenosis. The electrocardiogram demonstrated complete heart block (Fig. 1A) and the chest radiographs showed mild generalized cardiomegaly with marked calcification of the aortic valve. The serum electrolytes and SGOT were normal.

Corticotrophin (40 units b.i.d.) was commenced 8 days after admission in an attempt to restore sinus rhythm, but a week later, despite considerable general improvement and resolution of mild congestive cardiac failure, the heart block persisted. In view of this general improvement betamethasone $(0.5 \mathrm{mg}$. q.i.d.) was substituted and continued, with subsequent gradual withdrawal, for a further six weeks.

Three days after commencing corticotrophin steep inversion of the T waves was noted (Fig. 1B) and this electrocardiographic change persisted for two weeks after betamethasone had been withdrawn (Fig. 1C and D). No changes in heart rate, QRS complexes, systemic blood pressure, or serum electrolytes were observed during this time.

Case 2. This 56-year-old woman was known to have had coronary artery disease and essential hypertension for seven years before developing heart block. Complete A-V block (Fig. 2A) with left and right heart failure had been observed for 4 months before beginning steroid therapy. The SGOT at this time was 90 units $/ 100 \mathrm{ml}$. and the serum electrolytes were normal. Chest radiograph showed generalized cardiomegaly. 
Corticotrophin ( 40 units b.i.d.) was started and continued at this dose for $\mathbf{1 7}$ days. Despite resolution of the heart failure, reversion to sinus rhythm did not occur and corticotrophin was therefore gradually withdrawn over a further 10 days.

During this course of treatment progressive enlargement, inversion, and widening of the $T$ waves were observed in all the standard limb leads and in the V leads (Fig. 2B, C, and D). After withdrawal of corticotrophin these cardiographic changes reverted to the pre-steroid pattern (Fig. 2E). Throughout the treatment period the QRS complexes, heart rate, and blood pressure remained unchanged, and there was no disturbance in the serum electrolytes.

Case 3. This 58-year-old man was admitted to hospital in April 1963. He was known to have had progressive deterioration of his exercise tolerance for the 12 months before this hospital admission.

On examination he was in early congestive heart failure and was found to have bradycardia (40/min.). Chest radiograph showed generalized cardiomegaly and the electrocardiogram 2:1 A-V block with right bundle-branch block (Fig. 3A). The SGOT and serum electrolytes were normal.

Following rapid clinical deterioration while in hospital an attempt was made to restore sinus rhythm with steroids. Corticotrophin (40 units b.i.d.) was commenced 10 days after admission. Despite appreciable clinical improvement, no change in rhythm had been achieved a week later, and therapy was therefore gradually withdrawn over the next 10 days.

During this course of corticotrophin the $T$ waves and Q-T interval altered considerably without significant changes in either heart rate, QRS complexes, serum electrolytes, or systolic blood pressure (Fig. 3B, C). Five days after completing this treatment the electrocardiogram had returned to its pre-therapy pattern (Fig. 3D), but clinical improvement was maintained.

Case 4. This 80-year-old man was admitted to hospital in December 1963, having been found unconscious in the street. When first examined he had

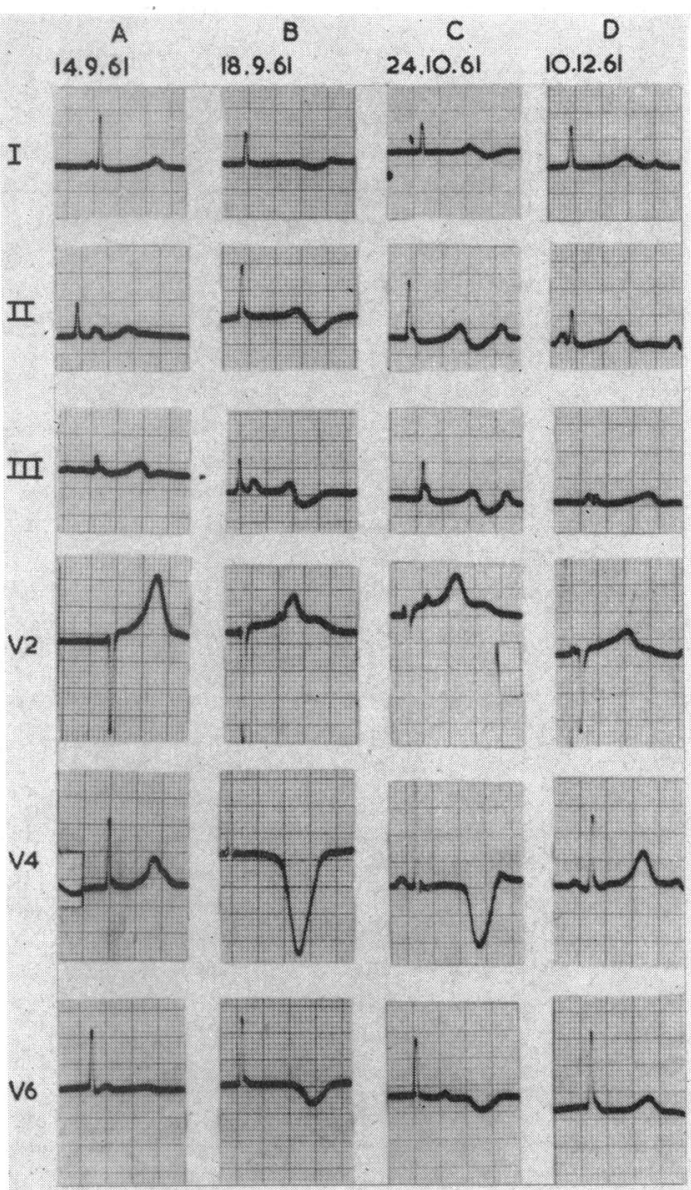

FIG. 1.-Case 1. (A) The QRS and T wave pattern before corticotrophin therapy. Complete A-V block with upright $\mathrm{T}$ waves in leads II, III, V2, V4, and V6. (B and C) Marked T wave inversion and prolongation of the Q-T interval in leads II, III, V4, and V6 during steroid therapy (ACTH and betamethazone). (D) Return of the $T$ wave configuration and $\mathrm{Q}-\mathrm{T}$ interval to the pre-steroid pattern two weeks after withdrawal of betamethazone. regained consciousness but had a right homonymous hemianopia and bradycardia of $46 / \mathrm{min}$. The electrocardiogram showed complete heart block (Fig. 4A) and the chest radiograph mild generalized cardiomegaly with gross pulmonary congestion. The SGOT was 235 units $/ 100 \mathrm{ml}$. and the serum electrolytes were normal.

Steroid therapy was commenced because of repeated severe Stokes-Adams syncope. Intravenous hydrocortisone (100 mg. 6-hourly) was given for the first 48 hours and was then replaced by prednisolone (10 mg. t.d.s.). After 48 hours his "blackouts" had ceased and his general progress was more satisfactory, though the heart block had persisted and the atrial rhythm had changed to fibrillation (Fig. 4B). Despite continuing steroid therapy for a further 32 days the A-V block and atrial fibrillation remained. During this period of therapy, however, considerable enlargement of the $T$ waves was observed (Fig. 4B and C). There was no significant alteration in the QRS complexes, heart rate, blood pressure, or serum electrolytes, and the $\mathrm{T}$ waves reverted towards the pre-treatment pattern as steroids were being withdrawn (Fig. 4D), 


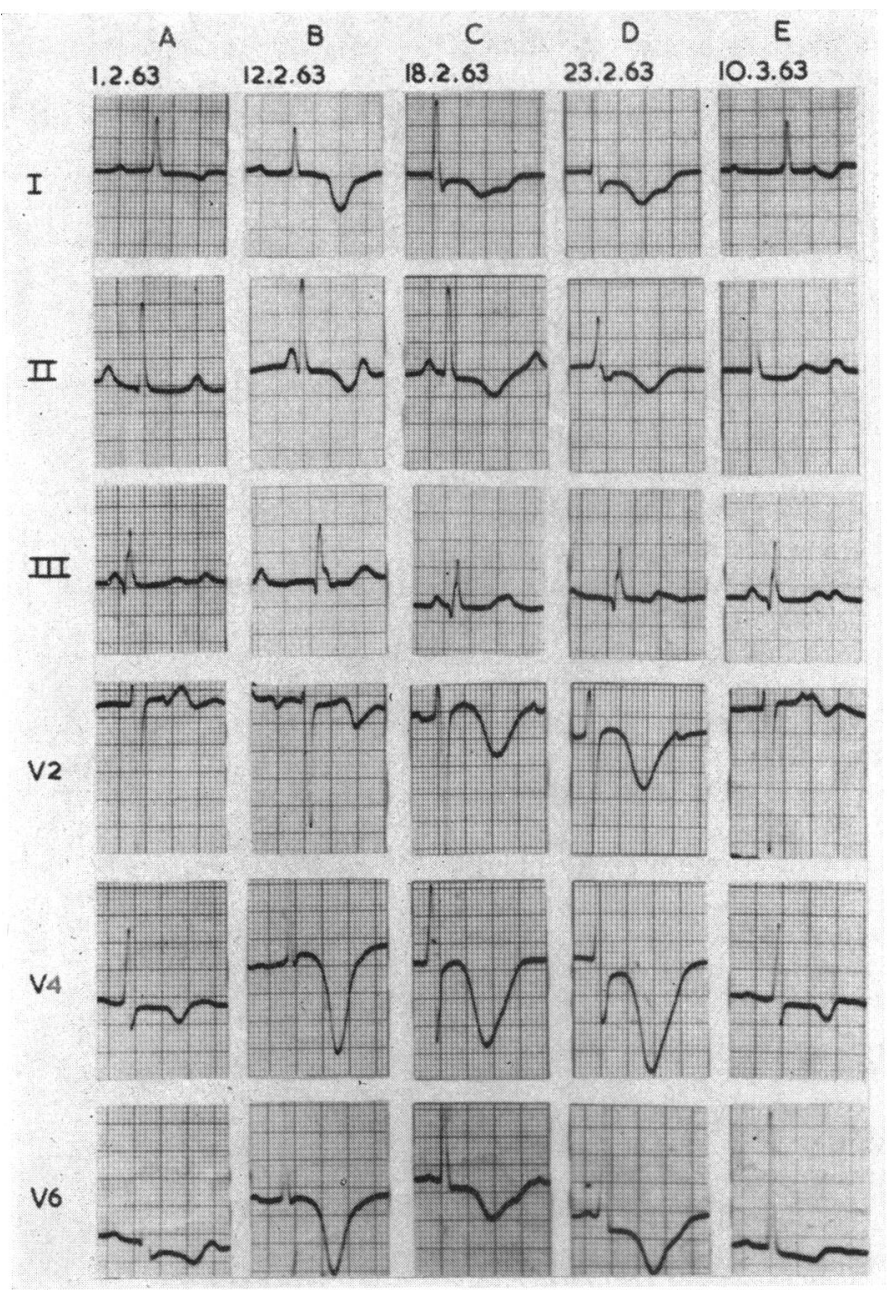

FIG. 2.-Case 2. (A) The QRS and T wave pattern before corticotrophin therapy. Complete A-V block with upright T waves in leads II, III, and V2, and inverted T waves in leads I, V4, and V6. (B, C, and D) Progressive enlargement, inversion, and widening of the T waves in leads I, V4, and V6 with inversion of T waves in leads II and V2. No changes in the QRS complex. (E) Return to the pre-therapy pattern two weeks after withdrawal of corticotrophin.

\section{DisCUSSION}

These observations show that, at least in the presence of bradycardia due to complete or partial A-V block, corticosteroids and corticotrophin can induce considerable changes in both the $T$ wave configuration and the Q-T interval in the electrocardiogram. These changes are reversible on withdrawal of steroid therapy and occur in the absence of significant alterations in heart rate, serum electrolytes, or systemic blood pressure. They appear to be independent of the type of steroid used and perhaps of the dose also, and occur without alterations in the QRS complexes.

All the factors that determine these electrocardiographic changes, however, are not obvious. Since in three patients (Cases 1, 2, and 4) the heart block was almost certainly due to coronary artery disease, with or without recent myocardial infarction (Case 4), focal ischæmic myocardial damage can be assumed to have been present. In the other patient (Case 3), however, the ætiology was less certain. 


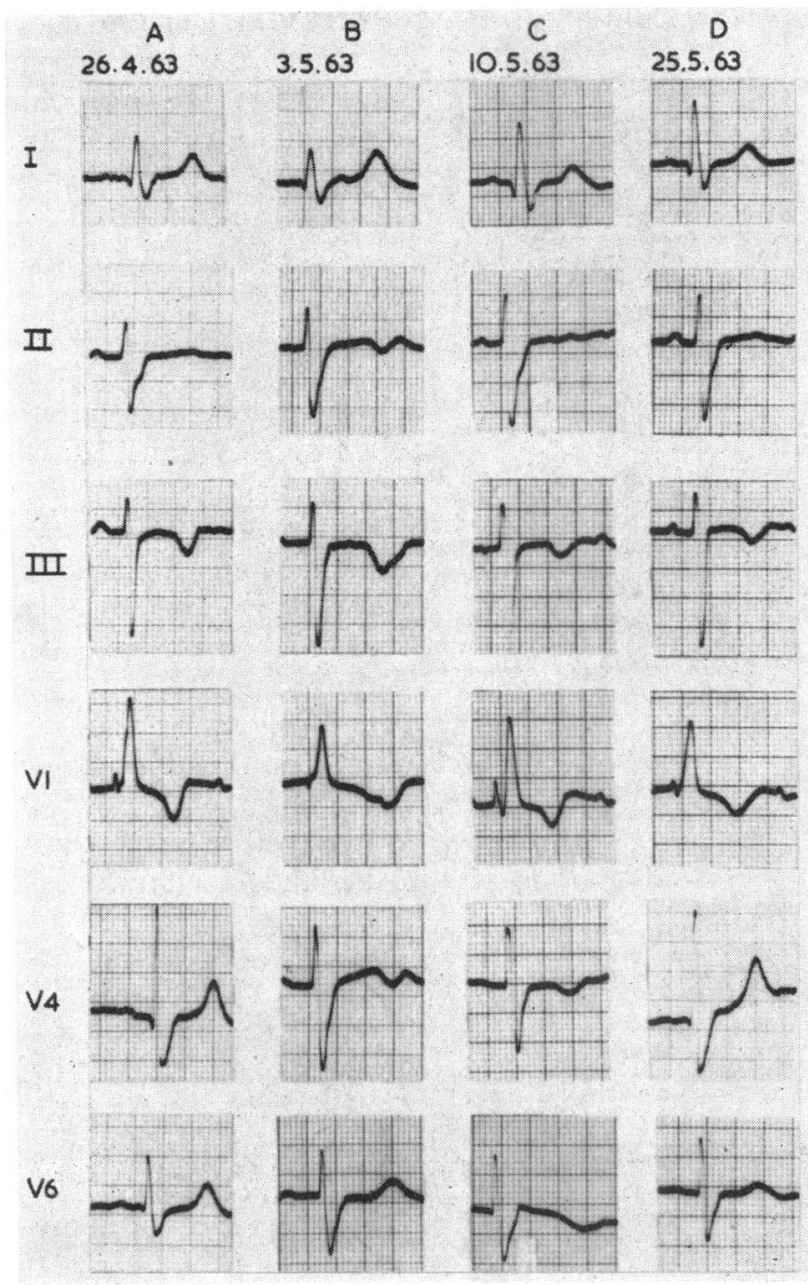

FIG. 3.-Case 3. (A) Two-to-one A-V block with right bundle-branch block before starting corticotrophin. (B and C) Prolongation of the $Q-T$ interval and changing $T$ wave configuration in leads V4 and V6 during corticotrophin therapy. (D) Return of upright T waves in leads V4 and V6 five days after completing the course of corticotrophin.

In each instance there was marked bradycardia, substantiating previous observations on the conditions necessary for giant T wave formation (Scherf, 1944; Ippolito et al., 1954; Szilagyi and Solomon, 1959). The duration of the block before beginning steroid therapy was, however, variable and acute myocardial damage does not, therefore, seem essential for the development of these steroid-induced disturbances of repolarization. Extension of the giant $T$ waves into adjacent leads (Case 2) suggests that, despite the absence of detectable serum electrolyte disturbance, ionic flux may be promoted in areas of "injured" muscle when the myocardium is exposed to an increased blood corticosteroid level. This in turn might be expected to interfere with the sodium-potassium pump across the cell membrane and so produce disturbance in repolarization at these sites.

It is not clear why these changes occurred in only 4 patients out of the 30 treated with steroids. The changes may have been masked by reversion to sinus rhythm in some patients (Aber and Wyn Jones, to be published), and in others the presence of widespread rather than focal ischæmic damage may have prevented their development (Pruitt, Klakeg, and Chapin, 1955). Certainly, we have never 


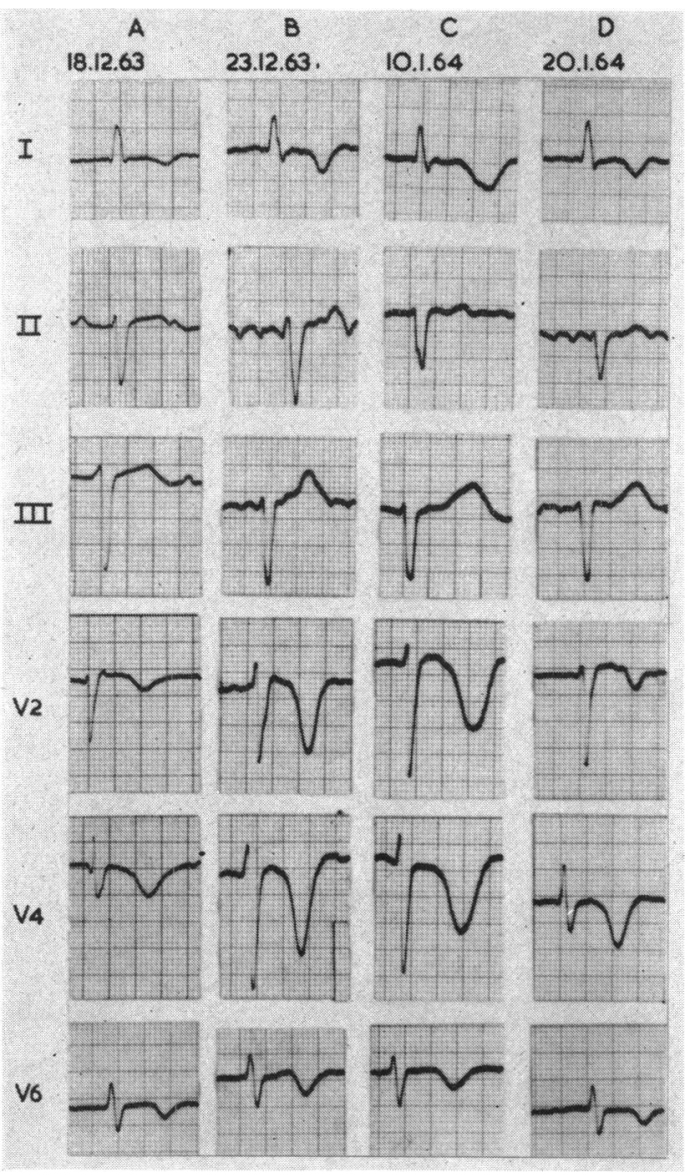

FIG. 4.-Case 4. (A) Complete A-V block showing pre-steroid QRS and $T$ wave configuration. (B) Atrial fibrillation with complete A-V block. Steep inversion of $T$ waves in leads $V 2$ and V4 four days after commencing steroid therapy (hydrocortisone and prednisolone). (C) Prolongation of the Q-T interval and further change in $T$ wave configuration while on prednisolone $(5 \mathrm{mg}$. q.i.d.). Atrial fibrillation persists. (D) Reversion of the Q-T interval and $T$ wave towards the presteroid pattern during gradual withdrawal of prednisolone therapy $(5 \mathrm{mg}$. daily). observed similar gross $T$ wave disturbances in the electrocardiograms of patients with generalized myocardial damage due to systemic lupus erythematosus, rheumatic fever, or other cardiomyopathies, when exposed to heavy steroid therapy.

Hypervolæmia during large doses of steroids could have caused increased diastolic loading and "stretching" of the myocardium. This seems unlikely, however, since the 3 patients (Cases 1, 2, and 3) who were in heart failure before therapy showed obvious and immediate clinical and radiological improvement.

Study of the effects of corticosteroids in experimentally induced heart block, with and without myocardial infarction, may help to solve some of the problems.

It is important to note that we observed no deterioration associated with these rather alarming electrocardiographic disturbances during the use of these large doses of steroids in heart block; indeed, the clinical benefit was most apparent (Aber and Wyn Jones, to be published).

\section{SUMMARY}

Evidence is presented that indicates that in the presence of bradycardia due to complete or partial A-V block, both corticotrophin and corticosteroids can sometimes induce considerable but reversible alteration in the $\mathrm{T}$ wave configuration and prolongation of the $\mathrm{Q}-\mathrm{T}$ interval in the electrocardiogram.

It is suggested that this perversion of myocardial repolarization results from local electrolyte flux and disturbances of the sodiumpotassium pump in areas of focal myocardial damage.

We should like to thank Dr. W. S. Sutton for permitting us to see and report Case 4.

\section{REFERENCES}

Aber, C. P., and Wyn Jones, E. (1960). Complete heart block treated with corticotrophin and corticosteroid. Brit. Heart J., 22, 723.

Garcia-Palmieri, M. R., Marchand, E. J., Diaz-Rivera, R. S., Santiago-Stevenson, D., and Rodriguez, H. F. (1956). The significance of giant negative $\mathrm{T}$ waves in coronary artery disease. Report of five cases. Amer. Heart J., 52, 521 .

Ippolito, T. L., Blier, J. S., and Fox, T. T. (1954). Massive T-wave inversion. Amer. Heart J., 48, 88.

Levine, S. A., and Brown, C. L. (1929). Coronary thrombosis: Its various clinical features. Medicine (Baltimore), $8,245$.

Pruitt, R. D., Klakeg, C. H., and Chapin, L. E. (1955). Certain clinical states and pathologic changes associated with deeply inverted $\mathrm{T}$ waves in the precordial electrocardiogram. Circulation, 11, 517. 
Scherf, D. (1944). Alterations in the form of the T waves with changes in heart rate. Amer. Heart J., 28, 332.

Szilagyi, N., and Solomon, S. L. (1959). Variations in the form of the T wave in a case of partial heart block. Amer. Heart J., 58, 637.

Wood, F. C., and Wolferth, C. C. (1934). Huge T-waves in precordial leads in cardiac infarction. Amer. Heart J., 9, 706.

\section{ADDENDUM}

Since submitting this paper for publication, we have seen 3 further examples of steroid-induced $T$ wave changes in heart block. One of these patients had marked hypokalæmia (serum potassium $2.0 \mathrm{mEq}$ per litre) at the time of these electrocardiographic abnormalities, which resolved on correction of this electrolytic disturbance despite continuation of corticotrophin therapy. 